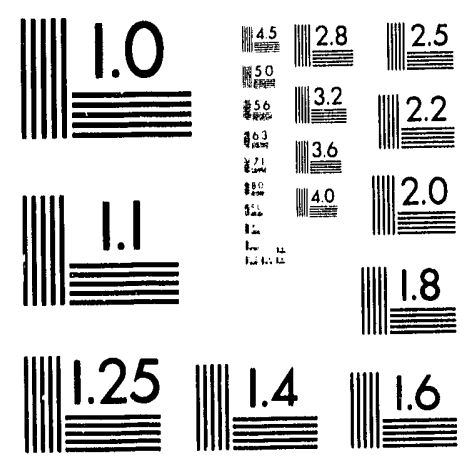



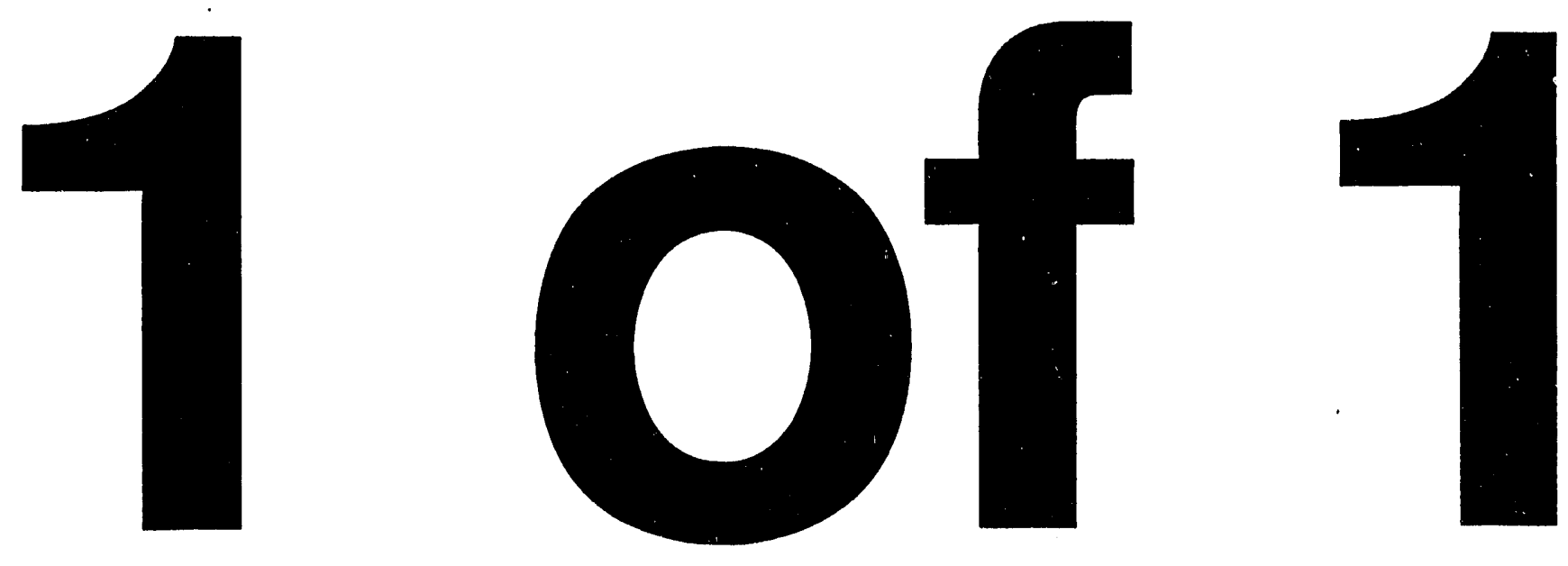


\title{
Conf-9309199--10
}

UCRL-JC-115005

PREPRINT

\section{Thermoelectric Cooled, Purge-and-Trap Device for Enhancing the Utility of Fiber Optic VOC Sensors}

\author{
E.A. Mizusawa \\ P.F. Daley \\ K.R. Kyle \\ K.C. Langry \\ S.M. Angel
}

This paper is prepared for SPIE

Chemical, Biochemical and

Environmental Sensors V Mtg.

Boston, MA, Septomber 7-10, 1993

August 1993

This is a preprint of a paper intended for publication in a journal or proceedings. Since changes may be made before publication, this preprint is made available with the understanding that it will not be cifed or reproduced without the permission of the author. 


\section{DISCLAIMER}

This document was prepared as an account of work sponsored by an agency of the United States Government. Neither the United States Government nor the University of California nor any of their employees, makes any warranty, express or implied, or assumes any legal liability or responsibility for the accuracy, completeness, or usefulness of any information, apparatus, product, or process disclosed, or represents that its use would not infringe privately owned rights. Reference herein to any specific commercial products, process, or service by trade name, trademark, manufacturer, or otherwise, does not necessarily constitute or imply its endorsement, recommendation, or favoring of the United States Government or the University of California. The views and opinions of authors expressed herein do not necessarily state or reflect those of the United States Government or the University of California, and shall not be used for advertising or product endorsement purposes. 


\title{
THERMOELECTRIC COOLED, PURGE-AND-TRAP DEVICE FOR ENHANCING THE UTILITY OF FIBER OPTIC VOC SENSORS
}

\author{
E.A. Mizusawa, ${ }^{1}$ P.F. Daley, ${ }^{2}$ K.R. Kyle, ${ }^{1}$ K.C. Langry ${ }^{1}$ and S.M. Angel ${ }^{1 *}$ \\ (1) Health and Ecological Assessment Division, (2) Environmental Restoration Division, Lawrence \\ Livermore National Laboratory, Livermore, CA 94550
}

\begin{abstract}
A thermoelectric cooled, purge-and-trap device (TEC) has been developed to enhance the utility of fiber optic VOC sensors. This simple device consists of a thin copper radiator which is sandwiched between two thermoelectric coolers. Two water-cooled, copper heat sinks are used to maintain the hot side surfaces of the thermoelectric coolers at 5 degrees $C$, allowing internal surface temperatures of the radiator to be controlled between 5 degrees $C$ to -43 degrees $C$. This paper describes the use of the TEC with a reversible, fiber optic, carbon tetrachloride sensor. The sensor by itself has a detection limit of around $1,000 \mathrm{ppmv}$ carbon tetrachloride in nitrogen. When the sensor is used with the TEC, vapor phase concentrations down to 100 ppmv can be measured.
\end{abstract}

\section{INTRODUCTION}

There are a number of research groups developing sensors for use in environmental monitoring and remediation of volatile organic compounds. Surface acoustic wave devices are being developed that detect changes in the viscoelastic properties of thin pu: 1 ier films as analytes are absorbed. ${ }^{1}$ A fiber optic spectrochemical emission sensor that can detect gas phase trichloroethylene, tetrachloroethylene, carbon tetrachloride and other related chlorinated compounds as low as one to five ppmv, has been used in the field at Hanford, Washington and Savannah River, South Carolina. ${ }^{2}$ A fiber optic based chemical sensor that can detect gas phase trichloroethylene from 0.2 to $500 \mathrm{ppmv}$, has been used to make remote measurements in wells and in the vadose zone using a cone penetrometer at Savannah River, South Carolina. ${ }^{3}$ This sensor has a tip which can be replenished with fresh colorimetric reagent using a dual syringe delivery/recovery system. ${ }^{4}$ Reversible, fiber optic based fluorescence sensors have been developed for real-time, in situ monitoring of volatile organic compounds. ${ }^{5-7}$ These sensors are based on the solvatochromic behavior of polymer-immobilized, fluorescent dyes, where absorption and emission properties of the dyes are dependent on the polarities of the solvents that are being absorbed by the polymer matrix. This paper describes the use of a thermoelectric cooled, purge-and trap device (TEC) to enhance the sensitivity of a reversible, fiber optic fluorescence sensor for carbon tetrachloride.

\section{EXPERIMENTAL SECTION}

\section{Sensor Design.}

The reversible, fiber optic, carbon tetrachloride sensor was made with a two meter length of silica core fiber having a diameter of 600 microns and a numerical aperture of 0.37 (Ensign-Bickford Optics Company, Avon, CT). One end of the fiher was terminated with a black Simplex Connector (AMP, Inc., Harrisburg, PA) using an epoxy mixture of Ardalite AW 136 High Strength Black Resin and HY 994 Hardener (Ciba Geigy, East Lansing, MI). Six inches of plastic buffer was stripped from the other end of the fiber, and the hard polymer cladding was removed with a flame. The exposed silica core was cleaned with methanol and cleaved. The freshly cleaved end was dipped in an silicone hexafluorine co-polymer elastomer mixture and allowed to cure overnight at room temperature (General Fiher Optics, Inc., Cedar Grove, NJ). The rigid, 750 micron polymer bead that formed on the end of the optical fiber was dipped in a concentrated Nile Red in carbon tetrachloride solution at 20 degrees $C$ for one second, and allowed to stand for 24 hours. 


\subsection{TEC Design.}

Figure 1 illustrates the basic design of the TEC. A 1.5-in $\times 1.5$-in $\times 0.125$-in copper radiator block was sandwiched between two MI1069T Thermoelectric Coolers that were connected in series (Marlow Industries, Inc., Dallas, TX). Two water-cooled, copper heat sinks were in contact with the hot side surfaces of the thermoelectric coolers. Dow Corning 340 Silicone Heat Sink Compound (Dow Corning, Midland, MI) was used between the radiator, thermoelectric coolers and heat sinks to maximize the thermal couple between the components. The TEC was placed in a dry, nitrogen filled chamber in order to avoid condensation from the atmosphere during the experiment.

Figure 2 illustrates the TEC radiator in detail. The TEC radiator was made by drilling a 0.1 -in diameter, U-shaped channel in the copper radiator block 1.125-in deep and 0.75-in across. Two 0.125-in copper tubes extended from the sample gas inlet and outlet ports of the radiator. The inlet port was packed with (0.01-in diameter copper wire to increase the internal surface area in the radiator. The fiber optic sensor was placed in the outlet port, with the sensor tip located at the hend of the U-channel. Two shut-off valves were placed in line so that the internal volume of the radiator could he isolated during the experiment. A hole was drilled in the radiator to accommodate an Omega 44(1)33 Precision Thermistor Element (Omega Engineering, Inc., Stamford, CT).

A schematic of the experimental setup is illustrated in Figure 3. A micrometer valve and a calibrated, gas flow meter were used to control the sample gas flow rate through the TEC. The internal surface temperature of the radiator was controlled with the two thermoelectric coolers and two copper heat sinks that were maintained at 5 degrees $C$ with a circulating water bath. When power to the thermoelectric coolers was off, the temperature of the system was 5 degrees $C$. When the thermoelectric coolers were given full power (21 V/3.6 A) from a Model 2050) Transistorized Power Sunnl $\because$, the internal surface temperature of the radiator dropped to -43 degrees $C$ (Power Design, Inc., New York, NY). Resistance output from the thermistor that was embedded in the radiator block was read with a Fluke 75 Multimeter (John Fluke Mfg. Co., Inc., Everett, WA). Fiber optic sensor emission was monitored with a portable, fiber fluorimeter that has been described in detail elsewhere. 6 For carbon tetrachloride measurements, a 50()$-\mathrm{nm}$ narrow band pass excitation filter, a 560-nm narrow band pass emission filter and a dicroic beam splitter were used. A 520-nm narrow band pass emission filter was used for reference measurements. Voltage output from the fluorimeter was collected with a Macintosh SE/30) Computer (Apple Computer Corp., Cupertino, CA) using Labview 2 Software (National Instruments Corp., Austin, TX).

\subsection{Reagents and Standards.}

Nile Red and HPLC grade carbon tetrachloride $(99.9+\%)$ were purchased from Aldrich Chemical Company, Milwaukee, WI, and used without further purification. Tenax GC 60/80 was purchased from Alltech Associates, Inc., Deerfield, IL. Nitrogen (Grade 5) was purchased from Airco Division, The BOC Group, Inc., Murray Hill, NJ, and the carbon tetrachloride standards (102, 514 and 1,020 ppmv in nitrogen) were obtained as a custom order from Byrne Specialty Gases, Inc., Seattle, WA.

\subsection{Procedures.}

Excitation and emission spectra in air and in the head space of neat carbon tetrachloride were taken in cork stoppered, 250-ml Erlenmeyer flasks with constant stirring at 2() degrees C. These spectra were collected on a custom, laboratory fiber optic fluorimeter that was comprised of SPEX 1680) Double Spectrometer excitation and emission monochrometers (SPEX Industries, Metuchen, NJ), a xenon arc lamp, housing and power supply (Oriel Corporation, Stratford, CT), a Model 126 Laboratory Photometer and Model 3150RF PMT Housing (Pacific Precision Instruments, Concord, CA), a Hamamatsu Photomultiplier Tube (Hamamatsu Photonics K.K., Toyooka Vill., Iwata-gun, Shizouka Pref., Japan) and a custom, fiber optic coupler (Spindler and Hoyer, Milford, MA). All other fluorescence measurements were collected on the portable, liher fluorimeter. 
Calibration curves for the reversible, fiber optic, carbon tetrachloride sensor by itself (no TEC) were generated at 5 degrees $C$ by standard addition of liquid carbon tetrachloride to a cork stoppered, 250-ml Erlenmeyer flask containing the sensor. The flask temperature was controlled with a water-filled, crystallizing dish that was equipped with a copper coil connected to a circulating water bath. Stir-bars were used in both the Erlenmeyer flask and crystallizing dish. Measurements were made three times to determine the sensor reproducibility.

For a typical run using the TEC and reversible, fiber optic, carbon tetrachloride sensor, the system was first purged for five minutes with sample gas flowing at $50 \mathrm{ml} / \mathrm{min}$ through the TEC which was at 5 degrees C. This established the experimental baseline. During the purge cycle, the 560-nm narrow band pass emission filter in the portable, fiber fluorimeter was replaced by a 520 -nm narrow band pass filter. The voltage output was recorded for one minute to monitor instrument drift during the experiment. After one minute, the filters were switched back to the original configuration. At the end of the five minute purge cycle, the sample gas flow was stopped and the thermoelectric coolers were turned on for five minutes, dropping the radiator temperature to -43 degrees $C$. This cooling was accompanied by a sharp drop in the sensor response. The sample gas flow was then allowed to resume for five minutes. The observed increase in sensor response during this cold cycle for the carbon tetrachloride standard gases $(0$, $1(12,514$ and $1,020 \mathrm{ppmv}$ ) were used to generate calibration curves. At the end of the cold cycle, the two shut off valves were closed, sample gas flow was stopped and the thermoelectric coolers were turned off. This isolated the carbon tetrachloride in the radiator at 5 degrees $C$. Calibration curves based on the magnitude of the sensor response in the valved off radiator at 5 degrees $C$ were generated. The entire procedure was repeated four more times to determine the reproducibility of the TEC/sensor system.

GC experimol"s were run to determine the trapping efficiency of the TEC. In a typical procedure, the TFC. radiator was first purged for one minute with sample gas flowing at $50 \mathrm{ml} / \mathrm{min}$. The sample gas flow was then stopped and the TEC was turned on for five minutes, dropping the radiator temperature to -43 degrees C. The sample gas flow was allowed to resume for five minutes. After tive minutes, the two shut-off valves were closed and the thermoelectric coolers were turned off, isolating the contents of the radiator at 5 degrees $C$. The outer lines were then purged with nitrogen gas for ten minutes at a flow rate of $10 \mathrm{ml} / \mathrm{min}$. A TENAX column placed at the outlet port of the radiator was used to trap the carbon tetrachloride isolated by the TEC, by opening the shut off valves which allowed the nitrogen gas to flow through the radiator. Standard gas injections of $0.25-\mathrm{ml}$ were used to calculate the trapping efficiency of the TEC, using a valve-to-valve free volume of $1.5-\mathrm{ml}$ and a radiator block free volume of $0.274-\mathrm{ml}$. These volumes were determined gravimetrically. GC experiments varying the sample gas flow times and rates were also run. For all GC experiments, a Model 850 Thermal Tube Desorber and a Model 851 Temperature Controller were used (Dynatherm Analytical Instruments, Cockeysville, MD) with an HP 5580A Series Gas Chromatograph equipped with a llame ionization detector (Hewlett Packard, Palo Alto, CA) and a DB 624 Column (J\&W Scientific, Folsom, CA).

\section{4, RESULTS AND DISCUSSION}

A reversible, fiber optic carbon tetrachloride sensor was specifically developed for use with the TEC. The sensor was made by immobilizing the fluorescent dye, Nile Red, on the end of an optical fiber with a silicone hexafluorine co-polymer elastomer. As shown in Figure 4, an emission maximum at 560-nm was produced in the presence of carbon tetrachloride vapor with 50()$-\mathrm{nm}$ excitation. The most important characteristic of this sensor was its extreme stability, generating reproducible baseline values through continuous temperature and high/low carbon tetachloride concentration cycles. Only one sensor was required for this study, which took place over a period of two months.

Figure 5 illustrates the calibration profiles of the sensor without the TEC at 5 degrees $C$, for () to 20,000) ppmv carbon tetrachloride in nitrogen. As illustrated in Figure 6, the sensor response was linear between the () and 20,000 ppmv range. The sensor by itself had a detection limit of around $1,000 \mathrm{ppmv}$. The sensor responded to vapor phase carbon tetrachloride concentrations above $20,0(0) \mathrm{ppmv}$, but went 
through some kind of reversible process at these high concentrations that required 30 minutes to regenerate the 0 ppmv baseline. Most likely the process was solvent swelling of the polymer tip, which temporarily changed the optical characteristics of the sensor. For practical purposes, the sensor by itself was found to be useful between 1,000 and 20,000$)$ ppmv range. To detect concentrations below 1,000 ppmv, the TEC had to be used.

Figure 7 and 8 show the full, expanded upper and expanded lower TEC/sensor profiles for 1,020 and 0 ppmv carbon tetrachloride in nitrogen, respectively. A complete cycle was 20 minutes long. Five cycles are shown for each gas sample to demonstrate the reproducibility of the method. During the first five minutes, purging the TEC with sample gas at 5 degrees $C$ established the sensor response baseline. Ideally the gas used to establish this haseline would be free of carbon tetrachloride. Since the device was being designed for remote measurements, we chose to establish the baseline with the gas sample that was being measured, eliminating the need to provide carbon tetrachloride free gas to the TEC. This process was possible because of the insensitivity of the sensor to vapor phase carbon tetrachloride concentrations below 1,(1)20 ppmv at 5 degrees $C$, relative to the signals obtained with the TEC. In the middle of the purge cycle, the sensor response with a 520 -nm narrow band pass emission filter was recorded to monitor instrument drift during the experiment. At the end of the purge cycle, the sample gas flow was stopped and the thermoelectric coolers were turned on, dropping the temperature of the radiator to -43 degrees $C$. The sensor signal, which is temperature dependent, dropped to around $2,(x)() \mathrm{mV}$ and leveled off. After five minutes of cooling, the sample gas was allowed to flow for five minutes. The sensor response during this time was used to generate the calibration curve in Figure 9, which is a plot of the rate at which the sensor response increased versus the carbon tetrachloride vapor phase concentration. The rate difference between 1,020 and 0 ppmv carbon tetrachloride can be seen by comparing the expanded lower TEC/sensor profiles in Figures 7 and 8.

After five minutes of sample gas flow, the two shut off valves were closed, the sample gas flow was stopped and the thermoelectric coolers were turned off. The sensor responded with a signal increase as the radiator warmed to 5 degrees $C$. When the sensor response leveled off, the magnitude of the signal relative to the sensor baseline value was recorded and used to generate the calibration curve shown in Figure 10. The upper expanded TEC/sensor profiles in Figures 7 and 8 show the signal differences between 1,020 and $0 \mathrm{ppmv}$ carbon tetrachloride. The shut off valves were then opened and the sample gas flow was turned on to begin purging the system for the next cycle. A reproducible spike was observed at the beginning of each purge cycle with () ppmv carbon tetrachloride. This is an inexplicable observation that occurs only with carbon tetrachloride free gas.

Vapor phase carbon tetrachloride concentrations down to 100 ppmv could be measured with the TEC/sensor system, even though GC results indicated that the trapping efficiency of the TEC was very low. For the experimental conditions of this study, a five fold increase in vapor phase carbon tetrachloride concentration was measured in the $1.5-\mathrm{ml}$, valve-to-valve free volume of the radiator block. Future work will include improving the trapping efficiency of the TEC.

\section{CONCLUSIONS}

A thermoelectric cooled, purge-and-trap device (TEC) has been developed to enhance the sensitivity of a reversible, fiher optic, carbon tetrachloride sensor. This sensor, which has a detection limit of around $1,()(X)$ ppmv carbon tetrachloride in nitrogen, can he used to measure vapor phase concentrations down to $1(0)$ ppmv when used with the TEC. Future work includes studying the effect of water vapor on the system, developing more sensitive fiber optic sensors for carbon tetrachloride and using the TEC with other fiber optic, VOC sensors.

\section{ACKNOWLEDGMENT}

This work was performed under the auspices of the U.S. Department of Energy by Lawrence Livermore National Laboratory (LLNL) under Contract \#W-74()5-ENG-48. The authors would like to express 
thanks to Caroline B. Purdy of the Office of Technology Development (OTD EM-542, TTP SF-221104) for supporting this research.

\section{REFERENCES}

1. G.C. Frye and S.J. Martin, "Utilization of Polymer Viscoelastic Properties to Enhance Acoustic Wave Sensor Performance," Proceedings of the Symposium on Chemical Sensors II, The Electrochemical Society, Honolulu, HI, 51-58, 1993.

2. N.C. Anheier, Jr., J.C. Evans, Jr. and K.B. Olsen, "Characterization and Monitoring of Total Organic Chloride Vapors," Presented at the US DOE Office of Environmental Restoration Information Exchange Meeting on Characterization, Monitoring and Sensor Technologies, Dallas, TX, 1992.

3. J. Rossabi, B. Colston, Jr., S. Brown, F. Milanovich and L.T. Lee, Jr., "In Situ Subsurface Monitoring of Vapor Phase TCE Using Fiber Optics," 3rd Int. Symp. on Field Screening Methods for Hazardous and Toxic Chemicals, Las Vegas, NV, 1993.

4. F.P. Milanovich, S.B. Brown and B.W. Colston, Jr., "Penetrometer Compatible Fiber Optic Sensor for Continuous Monitoring of Chlorinated Hydrocarbons - Field Test Results," Proceedings of the Symposium on Chemical Sensors II. The Electrochemical Society, Honolulu, HI, 643-647, 1993.

5. L.L. Blyler, Jr., R.A. Lieberman, L.G. Cohen, J.A. Ferrara and J.B. Macchesney, "Optical Fiber Chemical Sensors Utilizing Dye-Doped Silicone Polymer Cladding," Polyrner Engineering and Science, Vol. 29, No. 17, 1215-1218, 1989.

6. S.M. Barnard and D.R. Walt, "Fiher-Optic Organic Vapor Sensor," Environ. Sci. Technol., Vol. 25, No. 7, 1301-1304, 1991 .

7. S.M. Angel, B.L. Anderson and K. Langry, "Simple Reversible Fiber-Optic Chemical Sensors Using Solvatochromic Dyes," SPIE Chemical, Biochemical, and Environmental Fiber Sensors III, Vol. $1587,86-94,1991$. 


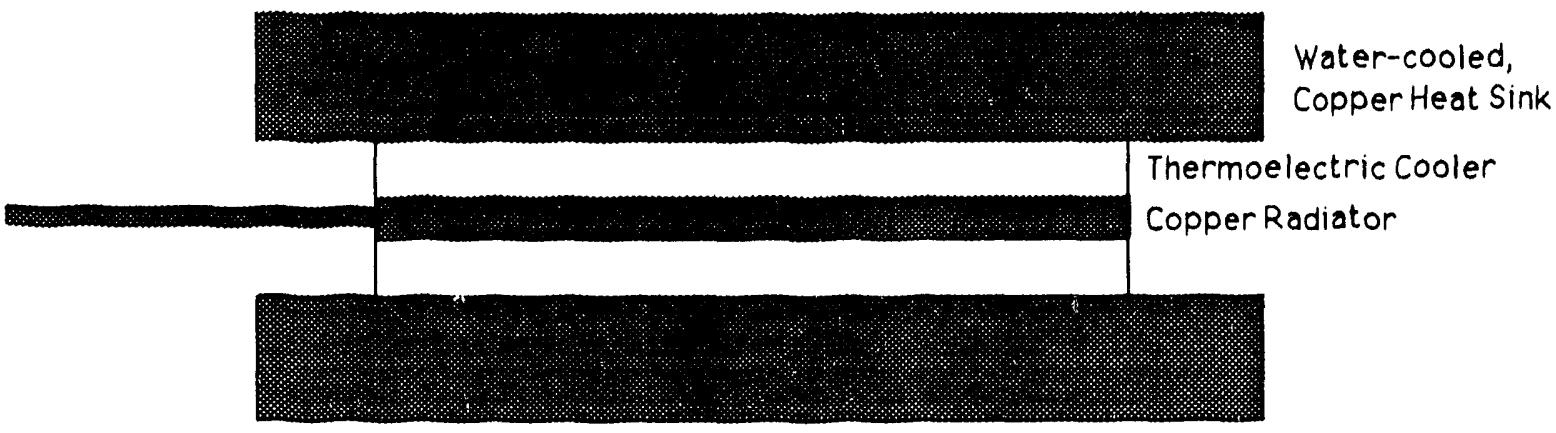

Figure 1. Basic Design of the TEC.

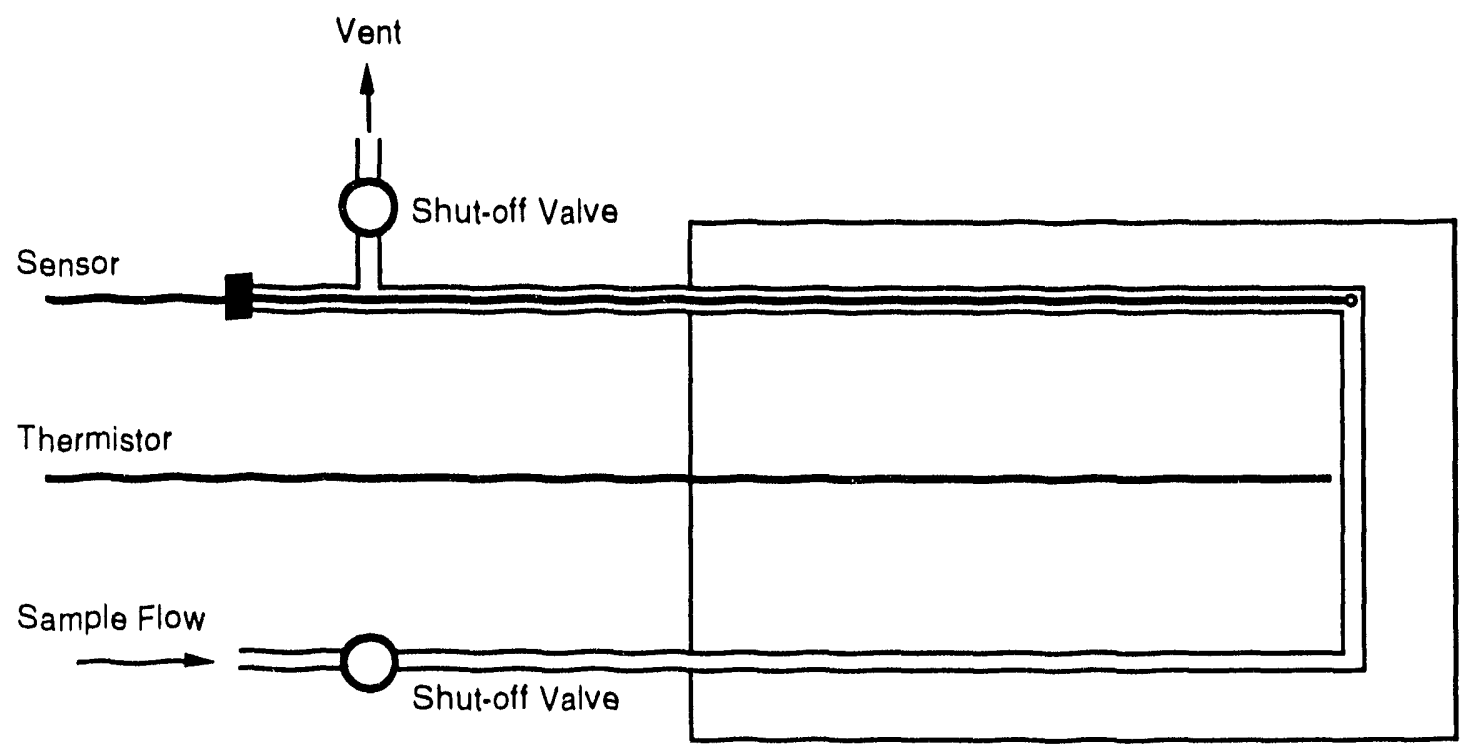

Figure 2. Schematic of the TEC Radiator. 


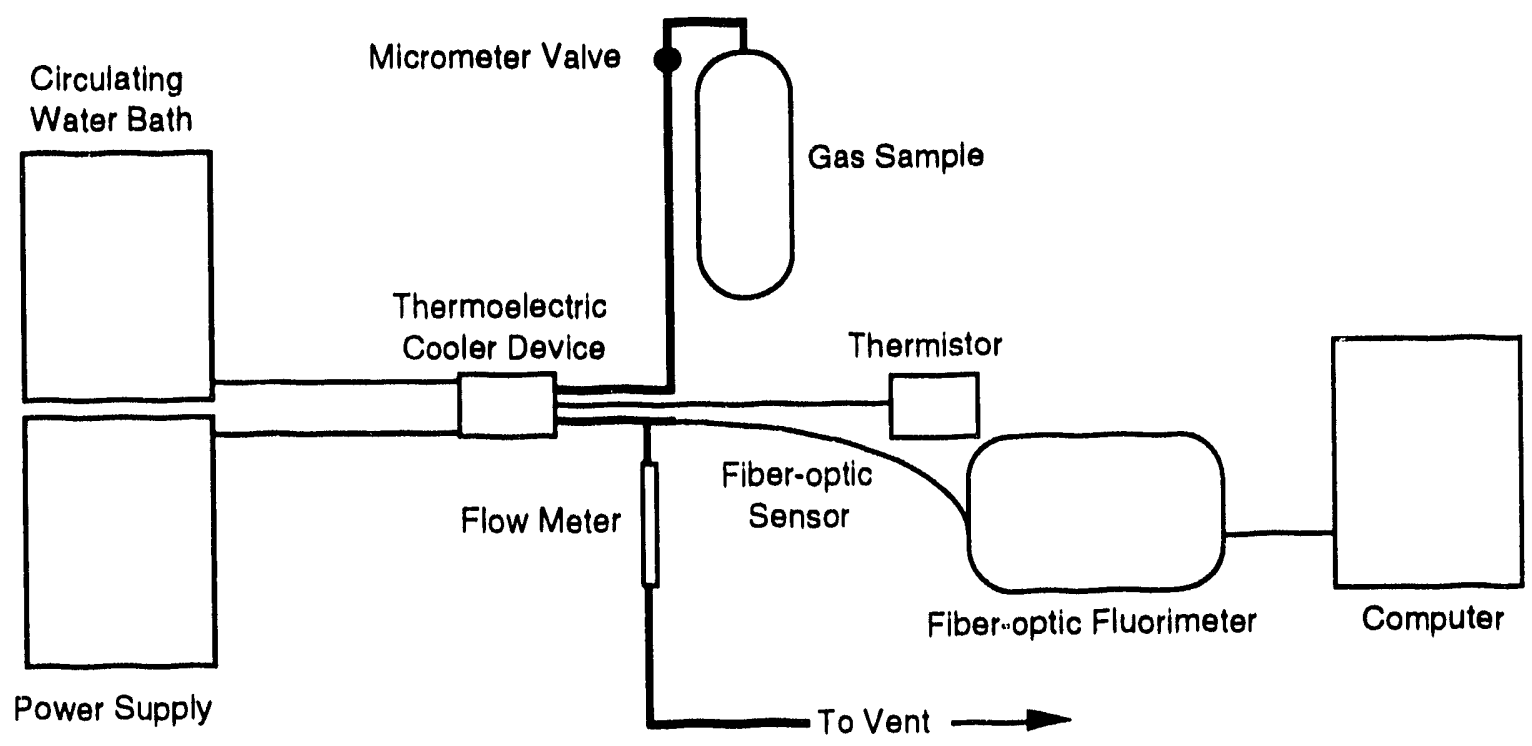

Figure 3. Schematic of the TEC Experimental Setup.

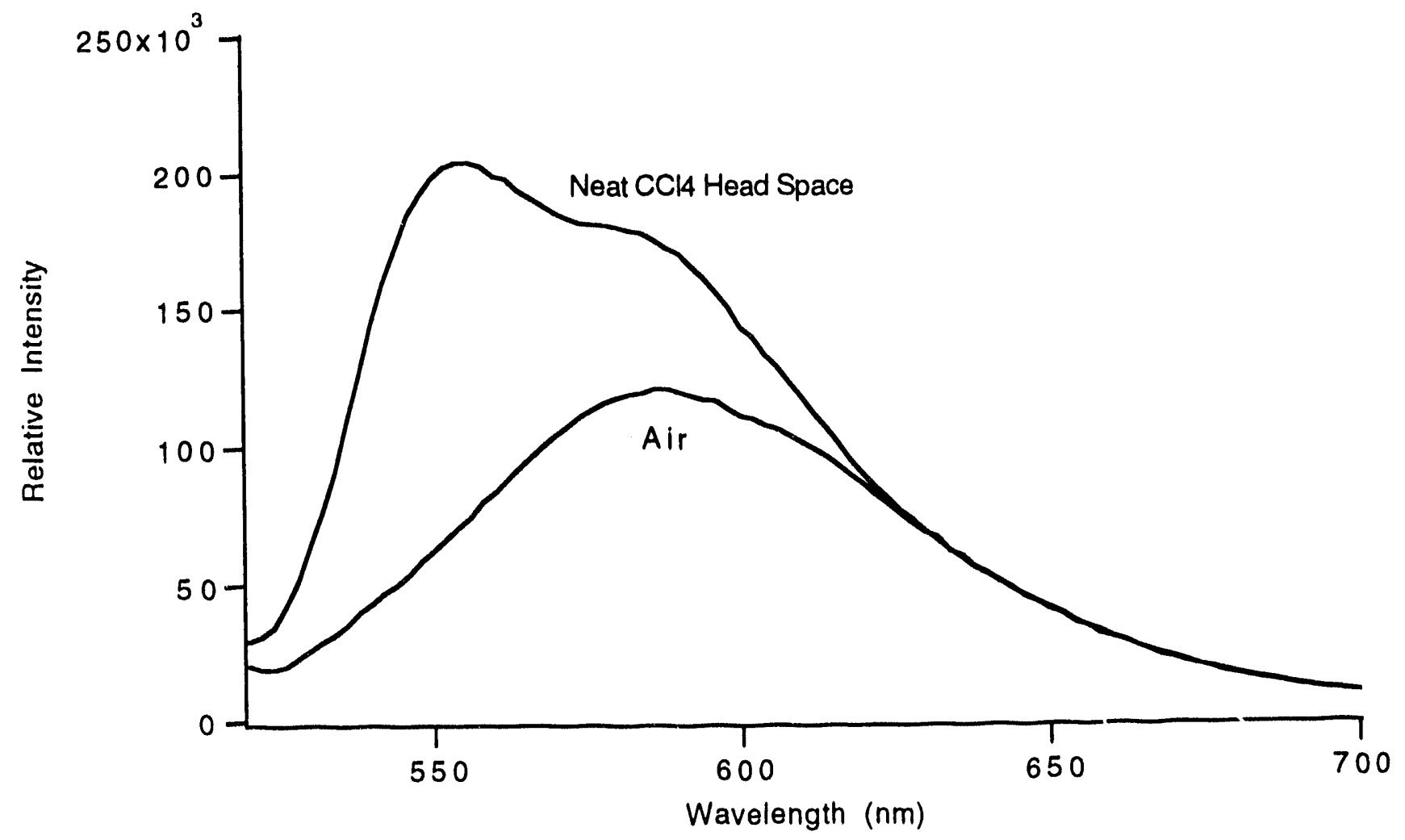

Figure 4. Nile Red/Gel Cladding Sensor Emission Spectra in Air and Head Space of Neat Carbon Tetrachloride (50) nm Excitation). 


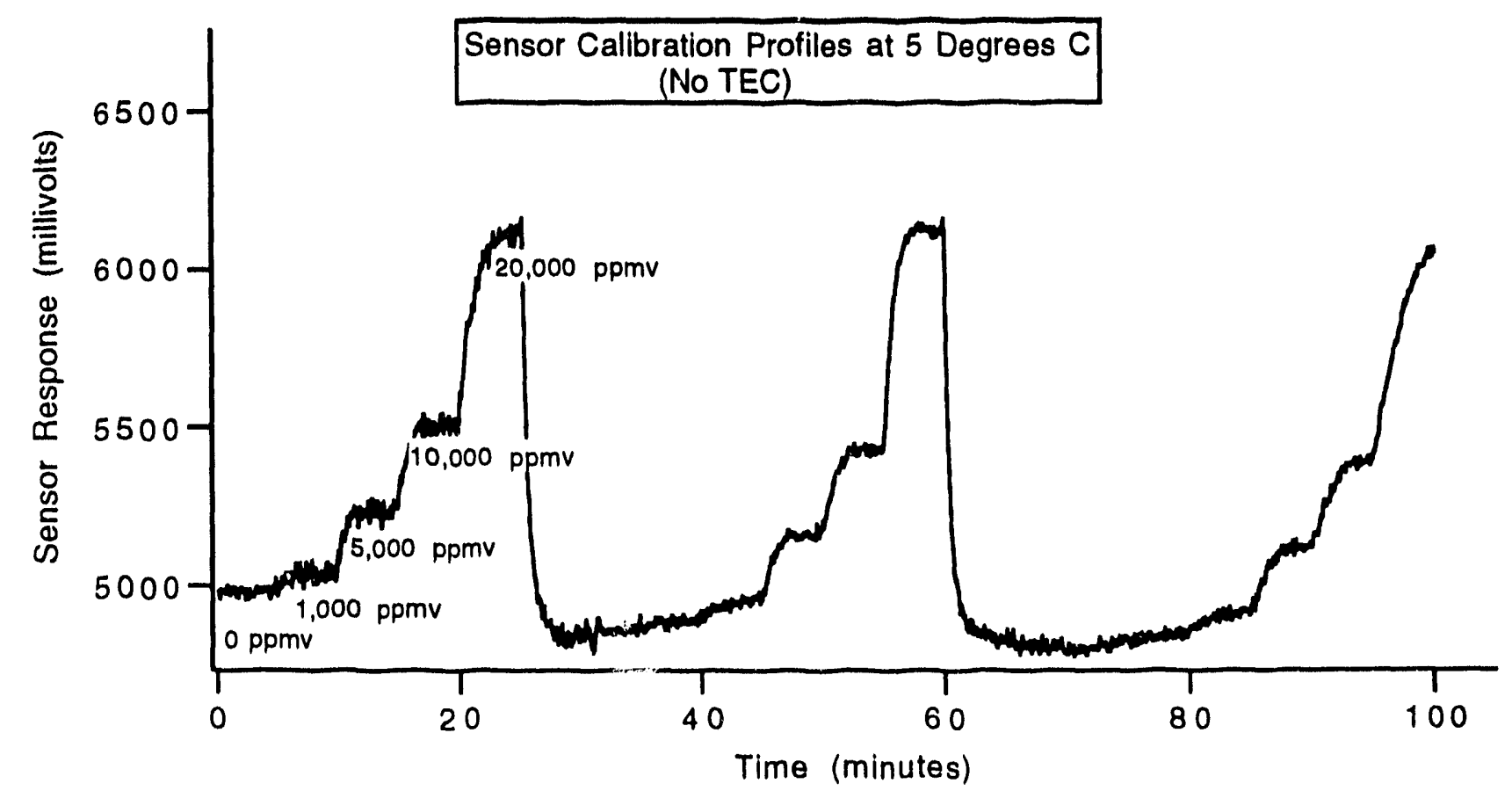

Figure 5. Sensor Calibration Profiles at 5 Degrees C (no TEC).

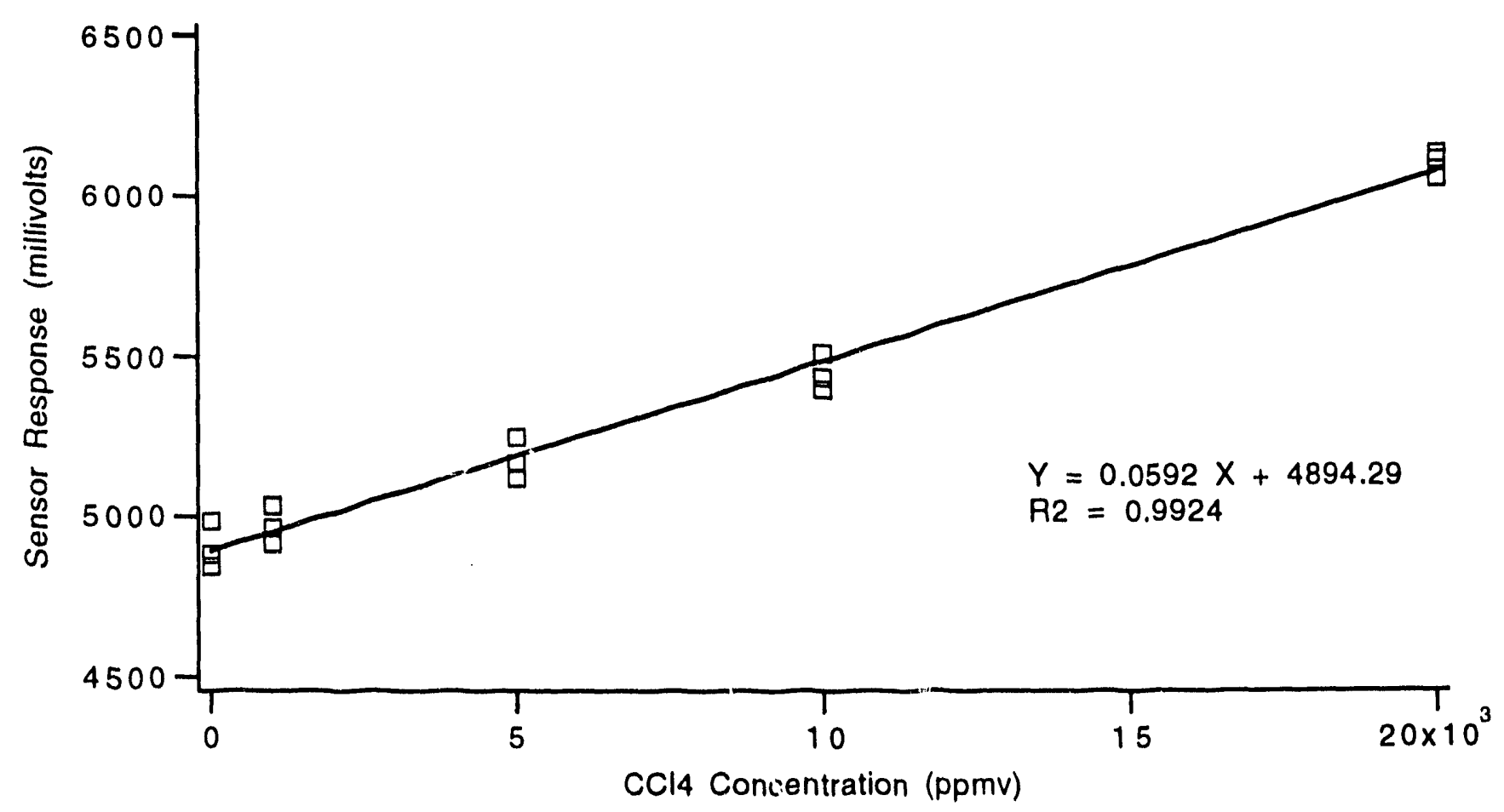

Figure 6. Calibration Curve For Sensor at 5 Degrees $C$ (no TEC). 

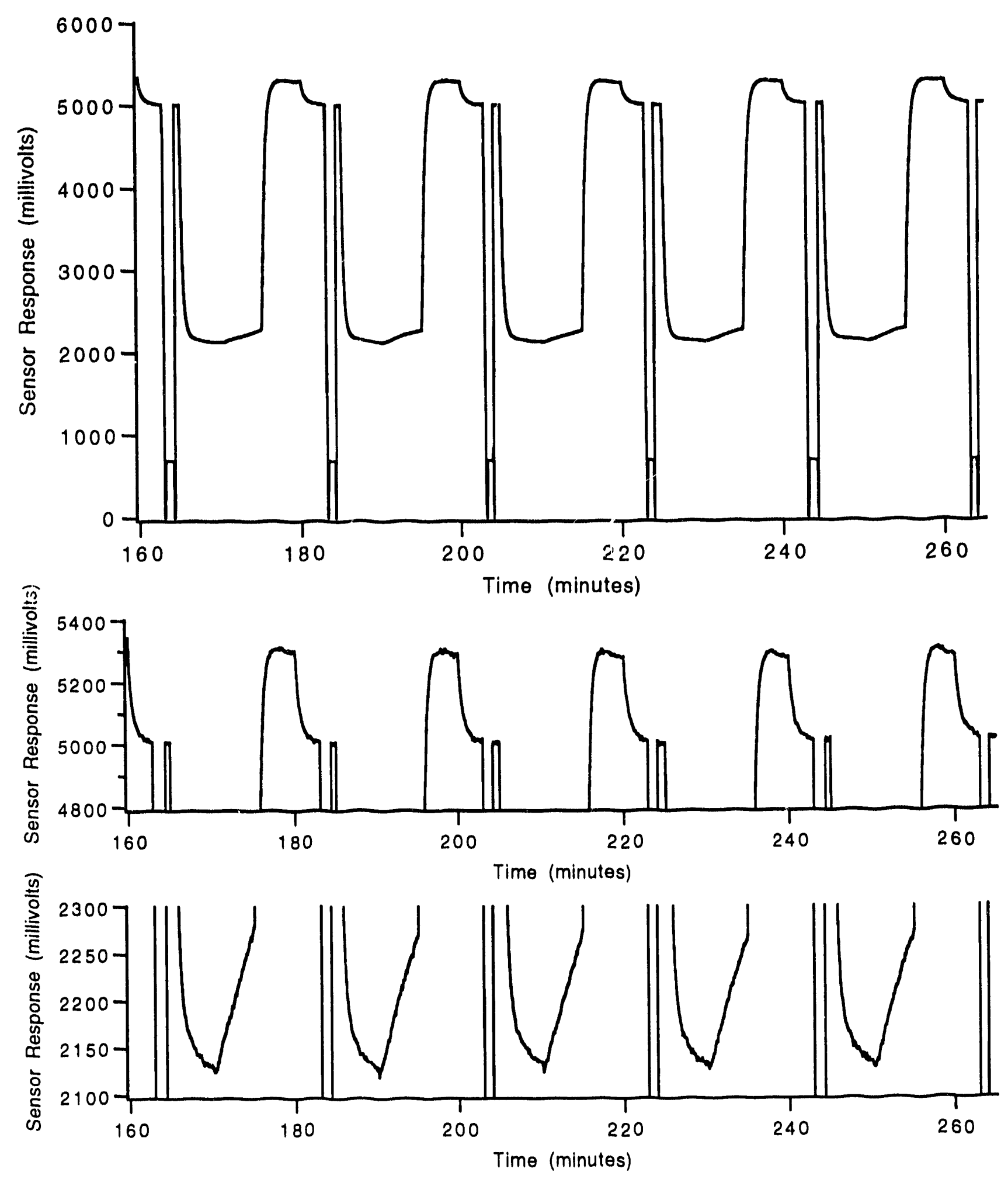

Figure 7. Full, Expanded Upper and Expanded Lower TEC/Sensor Profiles for 1,020 ppmv Carbon Tetrachloride. 

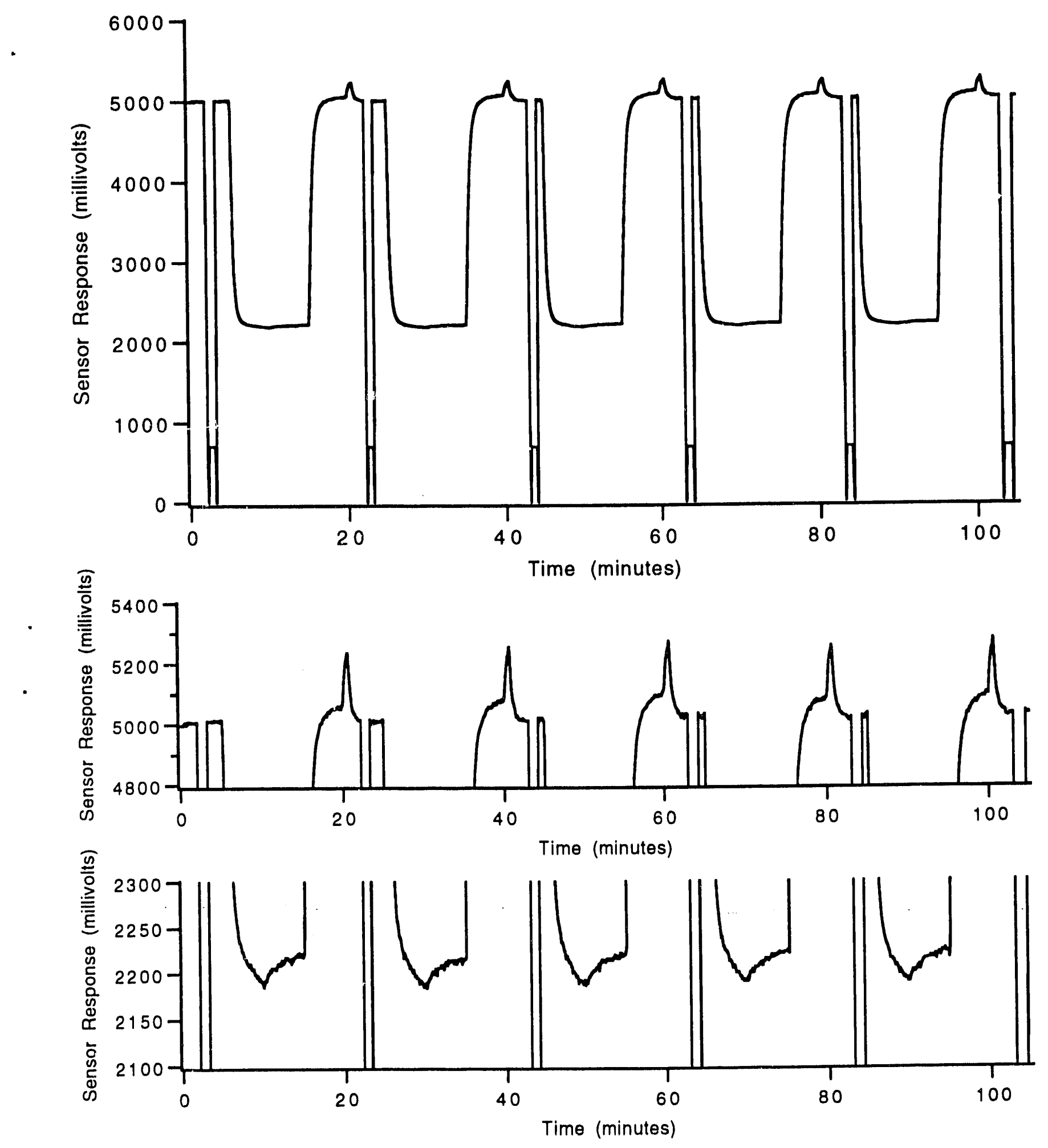

Figure 8. Full, Expanded Upper and Expanded Lower TEC/Sensor Profiles for 0 ppmv Carbon Tetrachloride. 


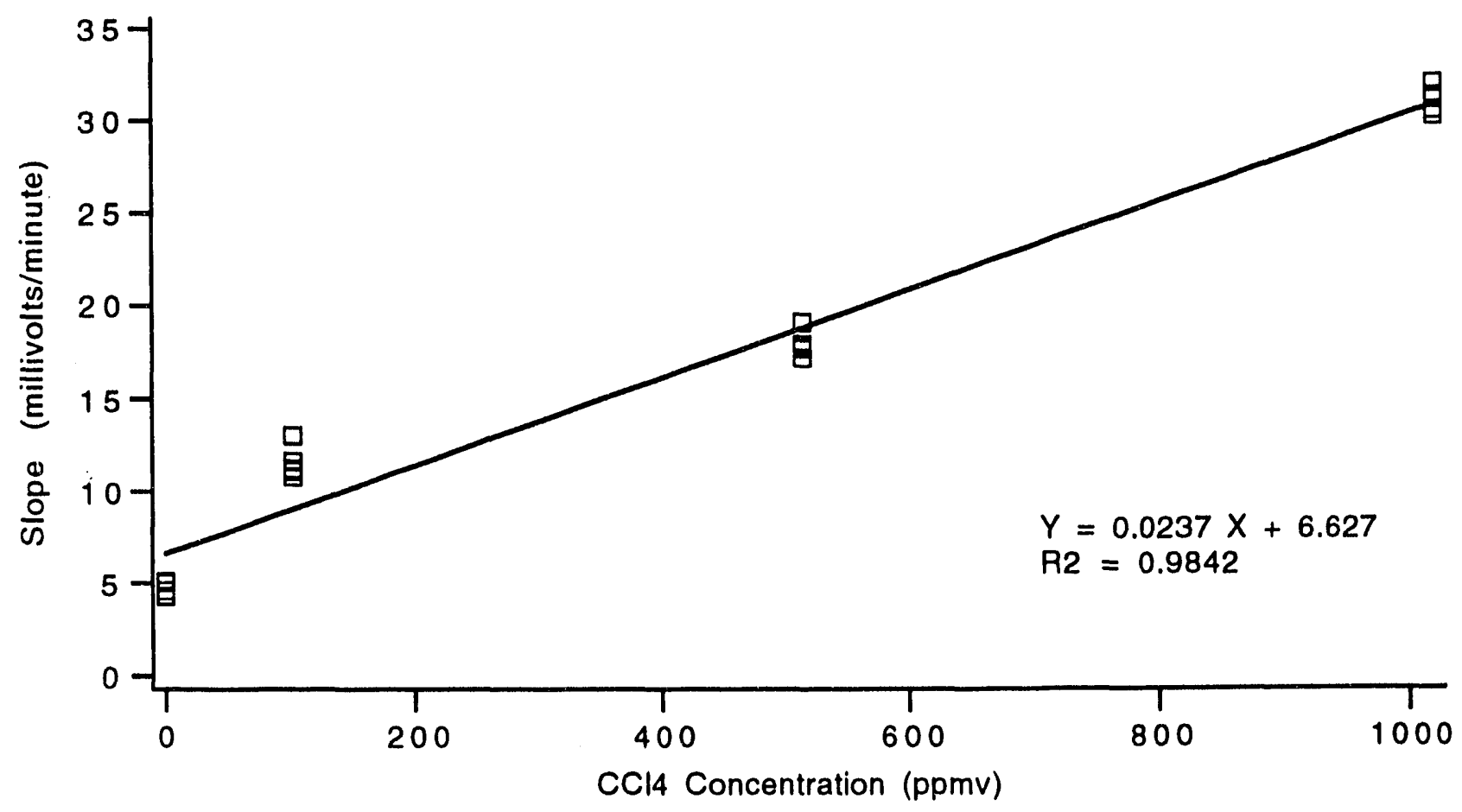

Figure 9. TEC/Sensor Calibration Curve Using Cold-cyc'e Slope Measurement.

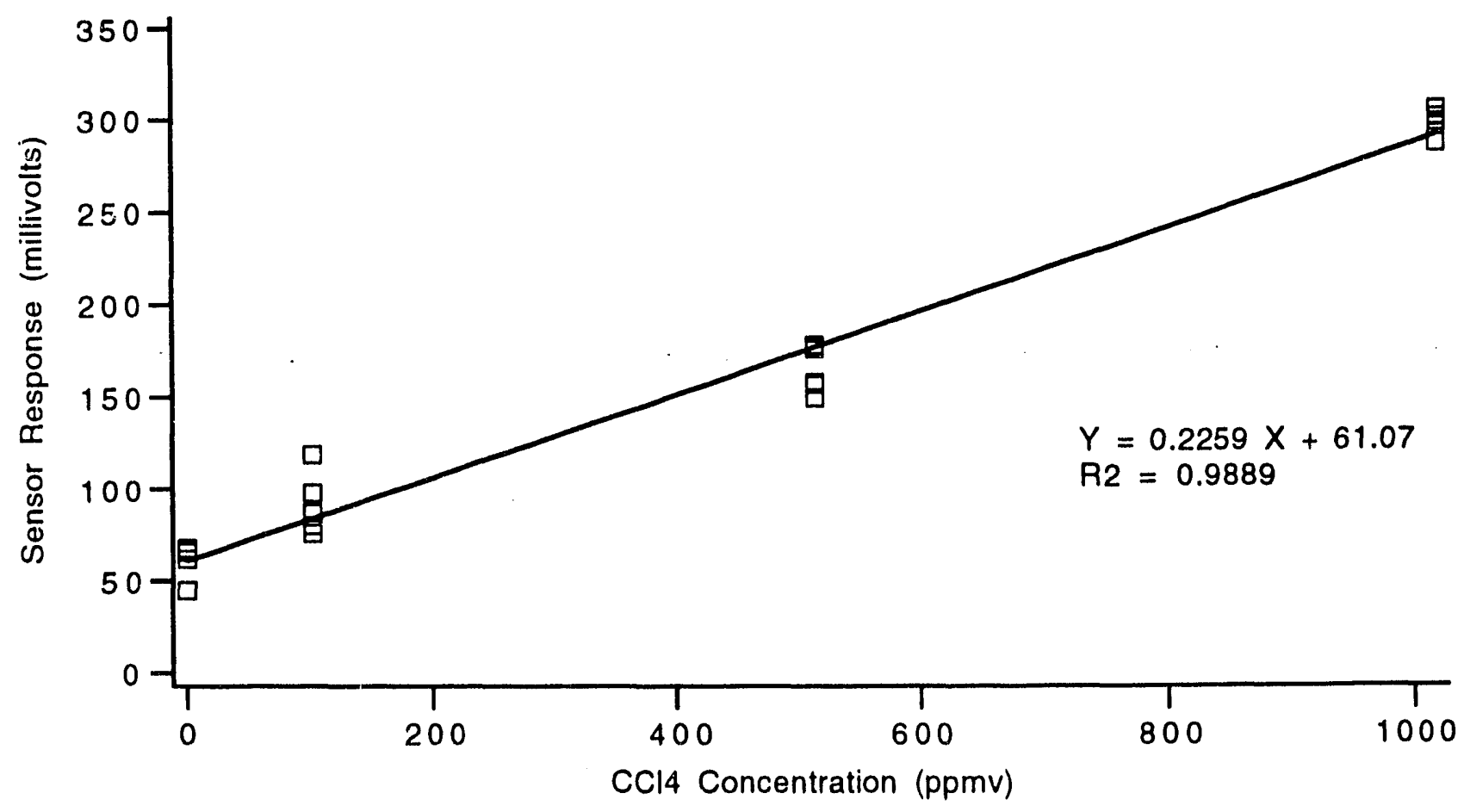

Figure 10. TEC/Sensor Calibration Curve Using Change in Sensor Response at 5 Degrees C. 

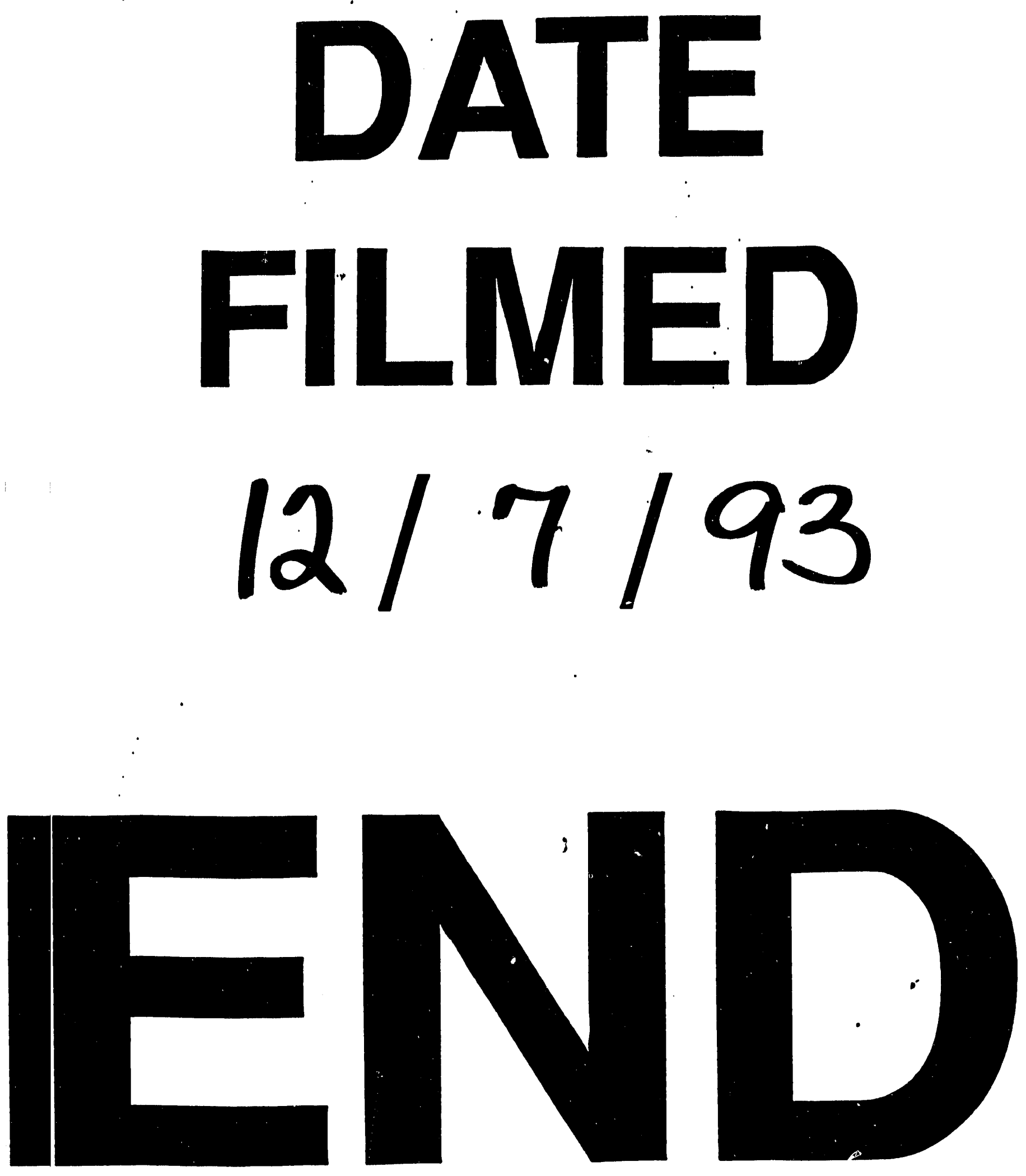
